15

\title{
Кулоновский взрыв дебаевского слоя как механизм формирования потока высокоэнергетичных ионов в плазме микропинчевого разряда
}

\author{
(C) А.Н. Долгов, ${ }^{1}$ Н.А. Клячин, ${ }^{2}$ Д.Е. Прохорович ${ }^{2}$ \\ ${ }^{1}$ Всероссийский научно-исследовательский институт автоматики им. Н.Л. Духова, \\ 127055 Москва, Россия \\ ${ }^{2}$ Национальный исследовательский ядерный университет „Московский инженерно-физический институт“, \\ 115409 Москва, Россия \\ e-mail: alnikdolgov@mail.ru
}

Поступило в Редакцию 5 декабря 2018 г.

В окончательной редакции 21 июля 2020 г.

Принято к публикации 14 сентября 2020 г.

\begin{abstract}
В низкоиндуктивной сильноточной вакуумной искре зафиксированы потоки ионов с энергиями порядка мегаэлектронвольт, распространяющиеся как в осевом, так и в перпендикулярном оси симметрии разряда направлении. Оценки, основанные на измерении полного заряда надтепловых электронов, испущенных из области перетяжки токового канала в осевом направлении, а также полного числа ионов указанных энергий, показывают, что зарегистрированные энергии ионов вполне соответствуют величине, достижимой в результате кулоновского взрыва дебаевского слоя перетяжки на стадии, предшествующей радиационному сжатию.
\end{abstract}

Ключевые слова: микропинч, кулоновский взрыв, дебаевский слой.

DOI: 10.21883/JTF.2021.03.50533.421-18

\section{Введение}

Ранее ускорительные процессы в пинчевом разряде рассматривались как результат воздействия на заряженные частицы генерируемого в перетяжке квазистатического электрического поля, например, резистивной природы $[1,2]$, и как результат формирования плазменного потока в процессе пинчевания [3]. Позднее была высказана гипотеза о том, что уход быстрых электронов из области перетяжки может приводить к созданию в ней положительного объемного заряда и затем - к кулоновскому взрыву [4]. Авторы работы [5] рассмотрели условия перехода электронов в режим непрерывного ускорения и получили оценки кинетической энергии ионов при кулоновском взрыве. Следует отметить, что некоторые допущения, которые они использовали в своих рассуждениях и оценках, не имеют достаточного обоснования. Во-первых, предполагается, что кулоновским является взрыв так называемой „горячей точки“ на заключительной стадии пинчевания плазмы в перетяжке. Во-вторых, предлагается принять, что кулоновский взрыв происходит приблизительно в то время, когда электростатическая энергия ионов, электрический заряд которых не скомпенсирован зарядом электронов, превысит магнитную энергию в объеме „горячей точки“.

Что касается наблюдаемого экспериментально взрыва „горячей точки“, то он не обязательно носит характер кулоновского, а вполне может происходить по причине аномального разогрева плазмы [6,7]. Комментарий ко второму допущению будет дан ниже.
В настоящей работе авторы представляют результаты выполненных ими экспериментов, которые могут свидетельствовать о реализации механизма кулоновского взрыва вследствие убегания ускоренных в осевом направлении электронов и образования области плазмы с избыточным положительным зарядом в дебаевском слое перетяжки как возможной причине формирования потока высокоэнергетичных ионов в сильноточном Z-пинче в среде тяжелых элементов.

\section{1. Схема эксперимента}

Объектом исследований являлись корпускулярные потоки, эмитируемые из плазмы разряда низкоиндуктивной сильноточной вакуумной искры, горящего в парах материалов электродной системы (рис. 1). В качестве материала электродов использовалась сталь марки Ст.3. Коаксиальная электродная система размещалась в откачиваемой камере при давлении остаточных газов не более $10^{-2} \mathrm{~Pa}$. Внутренний электрод 1 имел форму конуса с основанием диаметром $3 \mathrm{~mm}$ и углом раствора $60^{\circ}-90^{\circ}$, внешний 3 - цилиндра с плоским основанием и отверстием $\varnothing 3 \mathrm{~mm}$ на оси симметрии. Расстояние между электродами по оси составляет 5-6 mm. Разряд инициировался путем инжекции в межэлектродное пространство плазмы вспомогательного слаботочного разряда по поверхности диэлектрика 5, 6. В качестве источника тока использовалась батарея высоковольтных низкоиндуктивных конденсаторов 16, зарядное напряже- 


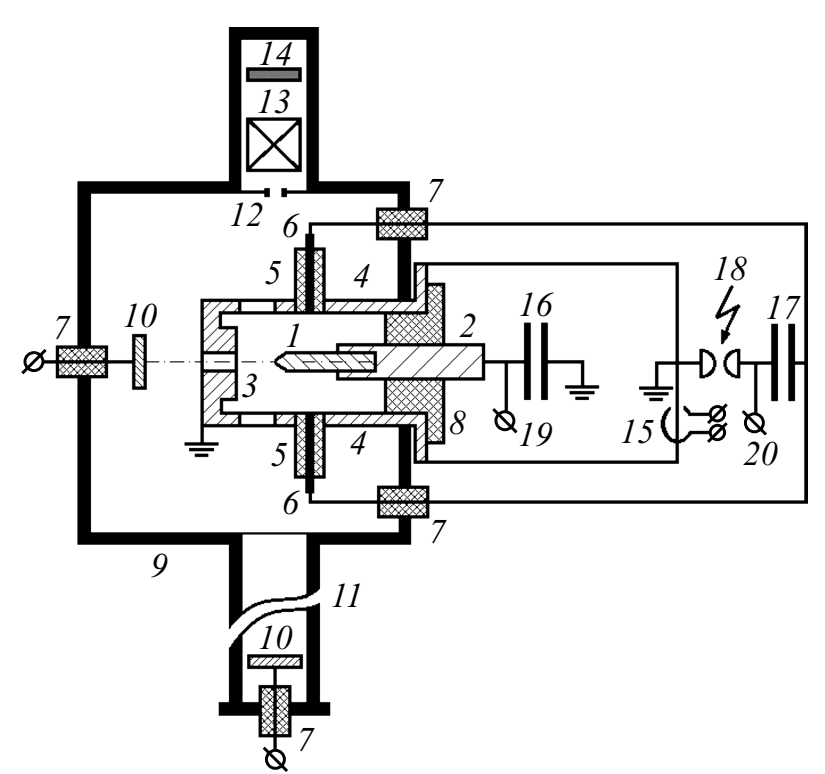

Рис. 1. Схема эксперимента: $1-$ внутренний электрод, 2 - внутренний токовод, 3 - внешний электрод, $4-$ внешний токовод, 5 - диэлектрическая (фторопласт) втулка, 6 - поджигающий электрод, 7 - проходной изолятор, 8 - разделительный изолятор, 9 - вакуумная камера, $10-$ коллектор заряженных частиц со смещенным потенциалом, 11 - пролетный тракт, 12 - магнитный экран со щелевой диафрагмой, 13 - параллельные плоские магниты, 14 трековый детектор, 15 - пояс Роговского, 16 - конденсаторная батарея в цепи основного разряда, 17 - конденсаторная батарея в цепи поджига, 18 - управляемый разрядник, 19, 20 - подключение источников высокого напряжения.

ние $-10 \mathrm{kV}$. Достигаемая сила тока $-140 \mathrm{kA}$, время нарастания тока $-1.5 \mu \mathrm{s}[8]$.

Отличительная черта схемы наших экспериментов состоит в том, что внутренний электрод является катодом. Следствием является распространение потока ускоренных в плазме разряда, а точнее в области перетяжки, электронов за пределы электродной системы в осевом направлении (рис. 2), что позволяет осуществить прямую регистрацию электронного потока.

Вакуумная камера имеет диагностические окна, которые позволяют наблюдать и исследовать с помощью различных диагностических средств процессы, протекающие в электроразрядном устройстве, как вдоль оси разряда, так и в направлении, перпендикулярном данной оси. Режим разряда контролировался путем регистрации тока поясом Роговского 15; динамики ВУФ и мягкого рентгеновского излучения, испускаемого плазмой разряда на стадии формирования микропинча, - c помощью вакуумного бипланарного фотодиода, который монтировался на фланце бокового окна вакуумной камеры; изображений, формируемых источниками излучения с длиной волны меньше $3-4 \AA,-$ с помощью рентгеновской камеры-обскуры, которая также монтировалась на фланце одного из боковых окон. Характерным при- знаком пинчевания плазменного канала разряда является наличие так называемой „особенности“ - резкого выброса на осциллограмме производной тока в районе прохождения ее через нулевую линию, т.е. вблизи максимума тока. Процесс формирования микропинча сопровождается всплеском ВУФ, мягкого рентгеновского излучения и образованием ярких источников излучения с длиной волны меньше $3-4 \AA-$ „горячих точек“ $[9,10]$.

Регистрация электронной эмиссии осуществлялась с помощью коллектора со смещенным потенциалом 10 , который размещался на оси симметрии разрядного устройства за пределами электродной системы на расстоянии примерно $10 \mathrm{~cm}$ от нее.

Регистрация ионной эмиссии осуществлялась в двух направлениях: осевом и радиальном, т.е. перпендикулярном по отношению к оси разряда, направлении. С этой целью на расстоянии $1.6 \mathrm{~m}$ от оси разряда (протяженность дрейфового пространства) на торце двух трубопроводов 11 , составляющих единую вакуумную систему с откачиваемой камерой, размещались два коллектора 10 со смещенным относительно заземленного корпуса 9 экспериментальной установки потенциалом. Энергия регистрируемых ионов измерялась с помощью времяпролетной методики [11]. В качестве реперных временных меток использовались следующие: на осциллограммах сигнала с коллектора ионов - фотопик, возникающий под действием ВУФ излучения разряда; на осциллограмме сигнала с пояса Роговского - „особенность“ тока разряда.

Дополнительно была использована методика регистрации потока ионов, распространяющегося в радиальном направлении, с помощью трекового детектора 14. В пределах откачиваемой камеры на расстоянии $15 \mathrm{~cm}$ от межэлектродного промежутка размещалась оптически прозрачная полимерная пленка CR-39, которая зареко-

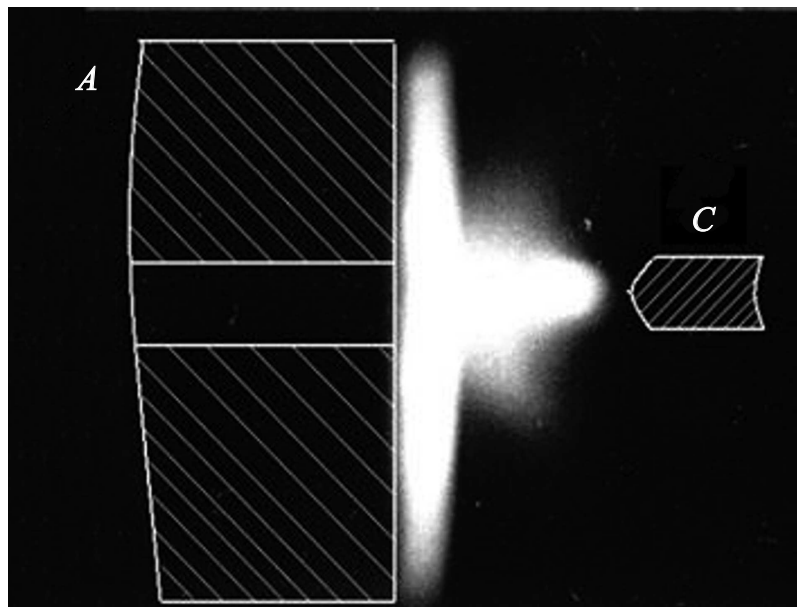

Рис. 2. Обскурограмма разряда низкоиндуктивной сильноточной вакуумной искры. Поток электронов, ускоренных в плазме разряда, распространяется в направлении анода и далее в пределах апертуры осевого отверстия в аноде за пределы электродной системы. 


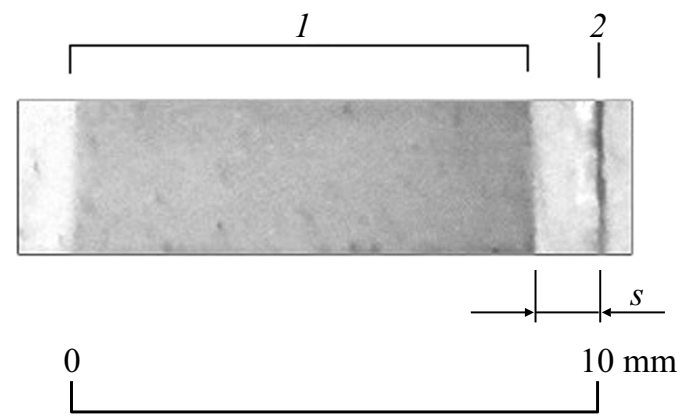

Рис. 3. След пучка высокоэнергетичных ионов на трековом детекторе в присутствии магнитного поля (обозначен цифрой 1). Черта справа - метка границы следа ионного пучка на детекторе в отсутствие магнитного поля (обозначена цифрой 2).

мендовала себя как эффективный трековый детектор при регистрации ионной эмиссии из плазмы низкоиндуктивной сильноточной вакуумной искры [12]. На поверхность пленки направлялся по нормали к ней коллимированный пучок ионов из разряда. Между детектором и коллиматором 12 располагалась область поперечного по отношению к направлению распространения ионного пучка и близкого к однородному по своей пространственной структуре магнитного поля индукцией $B=0.05$ Т и протяженностью $L=8 \mathrm{~cm}$, созданного парой постоянных плоских магнитов 13.

Измерение величины смещения ионного пучка в плоскости трекового детектора под действием приложенного магнитного поля позволило оценить величину энергии наиболее высокоэнергетичных ионов, эмитируемых из разряда. Подсчет плотности треков на поверхности детектора, оставленных регистрируемыми ионами, после их визуализации путем травления детектора в водном растворе щелочи вплоть до проявления максимального количества треков дал возможность измерить флюэнс ионов. С целью выявления величины смещения, вызванного магнитным полем, для наиболее высокоэнергетичных ионов была осуществлена визуализация следа ионного пучка на поверхности детектора в отсутствии магнитного поля следующим образом. Поверхность установленного в вакуумной камере детектора была освещена параллельным пучком света, проходящим по оси симметрии радиально расположенного окна вакуумной камеры и пересекающего область формирования ионного потока [8]. Край оптического изображения щели в коллимирующей диафрагме был очерчен на поверхности детектора (рис. 3).

\section{2. Результаты эксперимента}

Результаты прямой регистрации корпускулярной эмиссии с помощью коллектора со смещенным потенциалом $\varphi_{s}=-60 \mathrm{~V}$, размещенного на оси симметрии, но за пределами электродной системы, дали возможность оценить количество ускоренных и эмитированных из плазмы разряда электронов, след которых наблюдается в плазме разряда на рентгеновской обскурограмме, величиной $N_{e} \approx 10^{12}$ электронов.

По сигналу с радиально расположенного коллектора при потенциале смещения $\varphi_{s}=+40 \mathrm{~V}$ удалось зафиксировать группу ионов железа с энергией $\varepsilon_{i} \approx 3 \mathrm{MeV}$ (что отвечает максимуму тока на коллектор) в соответствии с временем пролета в дрейфовом пространстве. Аналогично по сигналу с коллектора, расположенного на оси разряда и имеющего такое же дрейфовое пространство, зафиксирована группа ионов с энергией $\varepsilon_{i} \approx 1 \mathrm{MeV}$.

Наблюдаемое на описанном выше трековом детекторе смещение наиболее высокоэнергетичной компоненты ионной эмиссии из разряда $s=1-1.5 \mathrm{~mm}$ (рис. 3), обусловленное воздействием приложенного магнитного поля, соответствует ожидаемой величине

$$
s \approx L^{2} /\left(2 r_{\mathrm{Li}}\right)
$$

при выполнении условия $s \ll r_{\mathrm{Li}}, \quad$ где $r_{\mathrm{Li}}=$ $=\left(2 M_{i} \cdot \varepsilon_{i}\right)^{1 / 2} /(Z \cdot e \cdot B)-$ ларморовский радиус для ионов массой $M_{i}$, при допущении, что заряд ионов составляет $Z \approx 10$, а энергия $\varepsilon_{i} \approx 3 \mathrm{MeV}$. Измерение плотности треков на детекторе и измерение полного заряда регистрируемых коллектором высокоэнергетичных ионов с учетом их зарядности в предположении изотропности разлета частиц в пределах телесного угла $4 \pi$ стерадиан позволило получить оценку полного числа высокоэнергетичных ионов на уровне $N_{i} \approx 10^{9}-10^{10}$.

\section{3. Обсуждение результатов эксперимента: стадия пинчевания, на которой происходит кулоновский взрыв}

Оценим приобретаемую кинетическую энергию $\varepsilon_{i}$, приходящуюся на частицу в результате „кулоновского“ разлета ионов из области перетяжки, получившей положительный заряд вследствие ухода ускоренных электронов. Вышеописанный коллектор, находящийся на оси разряда, регистрировал не только те убегающие из перетяжки вдоль оси токового канала ускоренные электроны, которые вносят вклад в формирование области нескомпенсированного положительного заряда, но также частицы, например, покидающие область разряда на его начальной стадии. Поэтому используем соотношение

$\varepsilon_{i} \cdot N_{i} \approx\left(e \cdot N_{e}^{(a c c)}\right)^{2} /\left(4 \pi \varepsilon_{0} \cdot r\right)=\left(e^{2} \cdot Z^{2} \cdot N_{i}^{2}\right) /\left(4 \pi \varepsilon_{0} \cdot r\right)$,

в котором $e-$ элементарный электрический заряд, $\varepsilon_{0}-$ диэлектрическая постоянная, $r-$ радиус перетяжки, $Z$ - средний заряд иона, $N_{e}^{(a c c)}-$ количество убегающих ускоренных электронов.

В дальнейших построениях мы будем опираться на представления и в некоторых случаях на расчетные 
параметры модели радиационного сжатия канала тока пинчевого разряда, которая, на наш взгляд, является наиболее последовательной и наиболее подтверждаемой материалами экспериментальных исследований [6,7,13-18]. Процесс формирования микропинча, согласно указанной модели, происходит в две стадии, т.е. канал тока претерпевает сначала первое, так называемое МГД сжатие, обусловленное преимущественно вытеканием вещества из области перетяжки, до радиуса примерно $r_{(1)} \sim 10^{-4} \mathrm{~m}$, а вслед за тем второе радиационное сжатие, обусловленное преимущественно излучательными потерями энергии, до радиуса порядка $r_{(2)} \sim 10^{-5} \mathrm{~m}$. Длина перетяжки принимается соизмеримой с еe радиусом. Именно во втором сжатии рождается ярко излучающий в диапазоне мягкого рентгена объект, называемый „горячая точка“. На стадии первого сжатия $Z_{(1)} \approx 6$, на стадии второго сжатия $Z_{(2)} \approx 20$ [6,19-21]. Численные оценки в случае „кулоновского взрыва“ на стадии первого сжатия дают величину $\varepsilon_{i}^{(1)} \sim 1 \mathrm{MeV}$ и $\varepsilon_{i}^{(2)} \sim 100 \mathrm{MeV}-1 \mathrm{GeV}$ для случая „кулоновского взрыва“ на стадии второго сжатия. Времяпролетная методика регистрации частиц не позволяла нам зафиксировать частицы с энергиями, соответствующими ситуации, когда „кулоновский взрыв“ осуществляется во втором сжатии.

Необходимо отметить, что в использованном нами полимерном детекторе треки частиц с энергиями $\varepsilon_{i}^{(1)} \sim 1 \mathrm{MeV}$ и $\varepsilon_{i}^{(2)} \sim 100 \mathrm{MeV}-1 \mathrm{GeV}$ после выполнения операции травления должны выглядеть существенно различным образом. А именно, треки частиц с энергиями $\varepsilon_{i}^{(1)} \sim 1 \mathrm{MeV}$ должны представлять собой лунки на поверхности детектора, радиус которых составляет порядка нескольких микрон и примерно равен их глубине. Напротив, для частиц с энергиями $\varepsilon_{i}^{(2)} \sim 100 \mathrm{MeV}-1 \mathrm{GeV}$ протравленный трек должен иметь протяженность, заметным образом превышающую поперечный размер [22,23]. В ряде экспериментов поверхность трекового детектора специально располагалась под углом $45^{\circ}$ по отношению к направлению падающего на нее потока частиц, что должно было облегчить выявление протяженных треков при наблюдении по нормали к поверхности детектора. Состояние поверхности и приповерхностного слоя трекового детектора было тщательно изучено с помощью оптического микроскопа. Протяженные треки не наблюдались.

Таким образом, свидетельств реализации явления кулоновского взрыва на стадии второго сжатия не обнаружено. В то же время регистрация потока частиц с энергиями $\sim 1 \mathrm{MeV}$, распространяющегося как в продольном, так и в поперечном по отношению к оси разряда направлении, может служить признаком реализации явления кулоновского взрыва на стадии первого сжатия. Некоторое различие в зарегистрированных энергиях частиц в потоках, распространяющихся в двух указанных направлениях, может объясняться более значительным торможением осевого потока в периферийной плазме разряда.
Следует признать, что большинство авторов придерживается мнения, согласно которому кулоновский взрыв происходит как раз на стадии после завершения второго сжатия, когда имеется аномальный разогрев плазмы в образовавшейся „горячей точке“. В результате „горячая точка“ взрывается, и в это время происходит генерация электронного пучка, жесткого рентгеновского излучения и ионов больших энергий, которые появляются с небольшой задержкой после основного импульса мягкого рентгеновского излучения [5,24-26]. Что касается самой возможности развития ускорительных процессов в перетяжке на стадии перехода от первого сжатия ко второму, то еще в работе [27] было показано как на основе теоретических оценок, так и при анализе экспериментальных данных о динамике спектра мягкого рентгеновского излучения микропинча, что резистивный механизм генерации ускоряющего электрического поля в перетяжке включается отнюдь не на финальной стадии второго сжатия, а уже по завершении первого сжатия за счет возникновения условий для раскачки колебаний в плазме перетяжки.

Далее приведем некоторые результаты, полученные в специально проведенной серии экспериментов. На рентгеновских обскурограммах области микропинчевания, регистрирующих изображение в спектральном диапазоне $\lambda<0.4 \mathrm{~nm}$, захватывающем $K$-спектр железа, можно наблюдать изображение следа пучка ускоренных электронов в периферийной плазме в отсутствие изображения „горячей точки“ (рис. 4). При этом изображения,

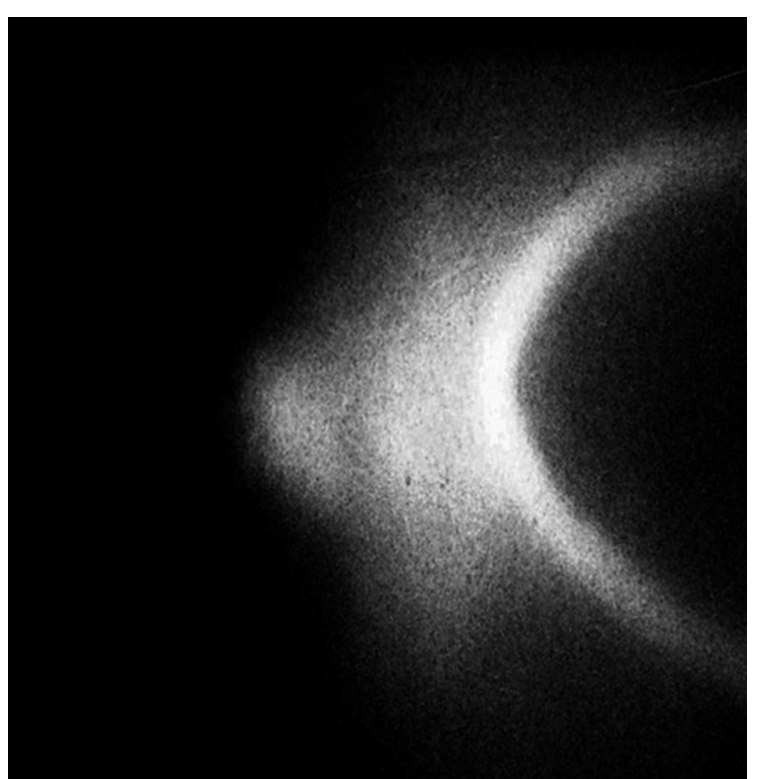

Рис. 4. Изображение электрода (в данном случае внутренний конический электрод выступает в роли анода, наблюдаемая сглаженная форма поверхности электрода - результат эрозии) и плазмы микропинчевого разряда в устройстве типа „сильноточная низкоиндуктивная вакуумная искра“, полученное в спектральном диапазоне $\lambda<0.4 \mathrm{~nm}$ в единичном разряде. Изображение „горячей точки“ отсутствует. 
одновременно полученные с помощью дополнительной камеры-обскуры в спектральном диапазоне $\lambda<1.5 \mathrm{~nm}$, захватывающем $L$-спектр железа, показывают формирование перетяжки в первом сжатии (рис. 5). Следовательно, развитие ускорительных процессов не связано напрямую с формированием, „горячей точки“. Более того, на изображениях, полученных с достаточно высоким пространственным разрешением в спектральном диапазоне $\lambda<1.5 \mathrm{~nm}$ за единичный разряд (рис. 6), можно наблюдать область взаимодействия убегающих из плазмы перетяжки вдоль ее оси электронов с окружающей плазмой за пределами перетяжки и область торможения убегающих электронов на поверхности электрода (анода).

Ранее было установлено, что уменьшая угол раствора конической поверхности внутреннего электрода удается в первом сжатии сформировать микропинч, обладающий при наблюдении в рентгеновской области спектра более высокой степенью осевой симметрии вдоль направления ускорения электронов, и это ведет к увеличению средней кинетической энергии электронов и соответствующему изменению регистрируемого в диапазоне энергий квантов $0.6-30 \mathrm{keV}$ спектра излучения, т. е. спектр становится заметно жестче [28].

Авторы настоящей работы проводили спектрополяриметрическое исследование рентгеновского излучения быстрого Z-пинча в среде тяжелых элементов в области характеристического спектра плазмообразующего элемента - железа [29]. Преимущественно продольная, т. е. вдоль оси симметрии, ориентация вектора электрического поля волны наблюдается для излучения $K_{\alpha}$-линии и сливающихся с ней линий ионов относительно низкой

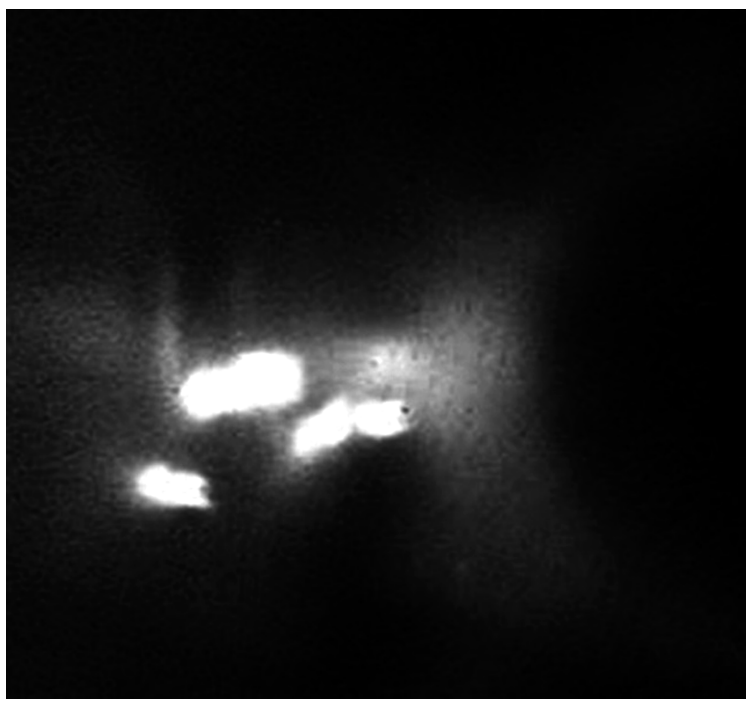

Рис. 5. Изображение электрода (внутренний электрод анод) и плазмы микропинчевого разряда в устройстве типа „сильноточная низкоиндуктивная вакуумная искра“, полученное в спектральном диапазоне $\lambda<1.5 \mathrm{~nm}$ в одном разряде с изображением, приведенном на рис. 4. Присутствуют изображения микропинчей, образовавшихся в результате первого сжатия.

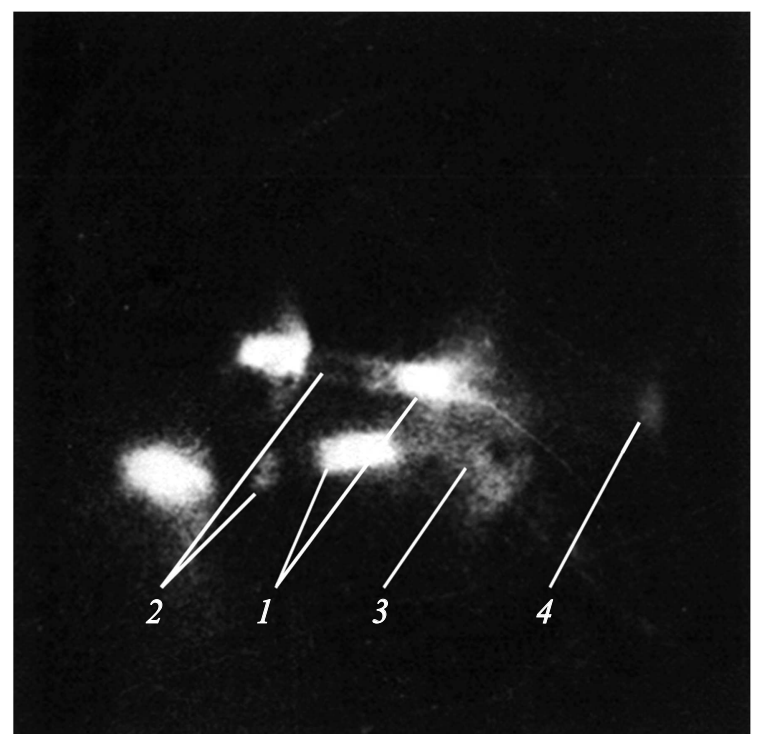

Рис. 6. Изображение объектов в микропинчевом разряде, излучающих в спектральном диапазоне $\lambda<1.5 \mathrm{~nm}$. Показаны: 1 - область формирования „горячей точки“, 2 - перетяжка, образовавшаяся после первого сжатия, 3 - область торможения убегающих из перетяжки электронов в плазме за пределами перетяжки, 4 - область торможения убегающих электронов на поверхности электрода.

кратности примерно до FeXVII включительно. Это говорит о том, что на стадии существования указанных ионов поляризация их излучения определяется продольным электрическим полем, присутствующим в плазме, и это поле по величине сравнимо с внутриатомным полем. Согласно модельным расчетам, подтверждаемым экспериментальными данными, ионы FeXVIII-FeXXII возникают в процессе достижения максимального сжатия плазмы в перетяжке $[6,10,29]$, т. е. во втором сжатии.

Квазистатическое электрическое поле резистивной природы оценим следующим образом:

$$
E_{\text {res }}^{(1)} \approx I \cdot R / r_{(1)} \sim 10^{8} \mathrm{~V} / \mathrm{m},
$$

где $R \approx 10^{-1} \Omega-$ сопротивление перетяжки, вычисленное с учетом потерь на излучение [30], $I \approx 10^{5} \mathrm{~A}-$ ток проводимости через перетяжку. Напряженность критического поля Драйсера, при превышении которого электроны начинают убегать, т.е. будут непрерывно ускоряться, оценим следующим образом [31]:

$$
E_{c r} \approx 0.214 \cdot \Lambda \cdot e /\left(4 \pi \varepsilon_{0} \cdot r_{D}^{2}\right) \approx 5 \cdot 10^{8} \mathrm{~V} / \mathrm{m},
$$

где $\Lambda \approx 15-$ кулоновский логарифм, $r_{D} \approx$ $\approx\left[\left(\varepsilon_{0} k T_{e}\right) /\left(e^{2} n_{e}\right)\right]^{1 / 2} \approx 10^{-8} \mathrm{~m}-$ радиус Дебая плазмы в перетяжке после первого сжатия, $k-$ постоянная Больцмана, $T_{e} \approx 50 \mathrm{eV}$ и $n_{e} \approx 10^{26} \mathrm{~m}^{-3}$ - электронная температура и концентрация плазмы в перетяжке после первого сжатия $[6,10,19,32]$. Сопоставление двух величин $E_{r e s}$ и $E_{c r}$ с учетом [31] свидетельствует о том, 
Данные эксперимента по регистрации потока заряженных частиц в осевом направлении

\begin{tabular}{c|c}
\hline \multicolumn{2}{c}{ Полное число ионов в перетяжке } \\
$N_{i \Sigma} \approx 10^{13}$ \\
\hline $\begin{array}{c}\text { Количество убегающих } \\
\text { ускоренных электронов, } \\
N_{e}^{(a c c)}\end{array}$ & Количество ионов, \\
вовлеченных в кулоновское \\
$10^{12}$ & ускорение, $N_{i \Sigma}$ \\
$10^{11}$ & $10^{13}$ \\
$10^{10}$ & $10^{11}$ \\
& $10^{9}$
\end{tabular}

что значительная часть электронов сможет переходить в режим непрерывного ускорения.

Таким образом, у нас появляются основания полагать, что в плазме перетяжки, образующейся в результате первого сжатия, действительно создаются условия для ускорения электронов в осевом направлении под действием электростатического поля, по-видимому, резистивной природы.

Оценим число ионов $N_{i}^{*}$, которые могут быть ускорены до зарегистрированных энергий в результате „кулоновского взрыва“" в зависимости от числа убегающих ускоренных электронов $N_{e}^{(a c c)}$, и полное число ионов $N_{i \Sigma}$ в перетяжке на стадии после первого сжатия исходя из того, что радиус перетяжки и ее длина сравнимы по величине следующим образом:

$$
\begin{gathered}
N_{i \Sigma} \approx n_{i} \cdot \pi r_{(1)}^{3}, \\
N_{i}^{*} \approx\left(e \cdot N_{e}^{(a c c)}\right)^{2} /\left(4 \pi \varepsilon_{0} \cdot r_{(1)} \cdot \varepsilon_{i}\right),
\end{gathered}
$$

где $n_{i} \approx 10^{25} \mathrm{~m}^{-3}$ - концентрация ионов в перетяжке на стадии первого сжатия [33,34]. Получим, что полное число ионов в перетяжке после 1-го сжатия составит $N_{i \Sigma} \sim 10^{13}$. Если мы примем $N_{e}^{(a c c)} \approx 10^{12}$, то оценка числа ионов, вовлеченных в „кулоновское“ ускорение, даст величину $N_{i}^{*} \sim 10^{13}$. Если принять $N_{e}^{(a c c)} \approx 10^{11}$, то $N_{i}^{*} \sim 10^{11}$. В случае $N_{e}^{(a c c)} \approx 10^{10}$ оценка даст $N_{i}^{*} \sim 10^{9}$. Таким образом, наиболее близкий к реально регистрируемой величине результат дает значение числа убегающих электронов, которые вносят вклад в формирование области избыточного положительного заряда, в интервале $N_{e}^{(a c c)} \approx 10^{10}-10^{11}$, что заведомо не превышает данные эксперимента по регистрации потока заряженных частиц в осевом направлении (см. таблицу).

\section{4. Предлагаемая модель - кулоновский взрыв дебаевского слоя}

В качестве причины кулоновского взрыва мы рассматриваем нарушение зарядовой нейтральности плазмы перетяжки вследствие убегания ускоренных в осевом направлении электронов и образования области плазмы с избыточным положительным зарядом по окончании первого сжатия. Однако убегание электронов под действием электростатического электрического поля резистивной природы становится возможным только в приосевой области, радиус которой приближается к величине ларморовского радиуса электрона на границе этой области [35]:

$$
r^{(a c c)}=r_{(1)} \cdot\left[2 \pi\left(2 m_{e} \cdot \varepsilon_{e}\right)^{1 / 2} /\left(\mu_{0} e \cdot I\right)\right]^{1 / 2} \approx 3 \cdot 10^{-6} \mathrm{~m},
$$

где $m_{e} \approx 10^{-30} \mathrm{~kg}$ - масса электрона, $\varepsilon_{e} \approx 50 \mathrm{eV}-$ средняя тепловая энергия электронов в перетяжке на стадии первого сжатия [6,32]. В этой же области должен был бы образоваться и избыточный положительный заряд, но тогда мы наблюдали бы среди регистрируемых высокоэнергетичных ионов частицы с энергией, по крайней мере на порядок более высокой по сравнению с той, что реально наблюдается. Отсутствие таковых заставляет предположить следующий механизм формирования области плазмы с избыточным положительным зарядом. Нетрудно убедиться, что на стадии первого сжатия выполняются следующие условия:

$$
\begin{gathered}
r_{\mathrm{Le}}^{(\text {extr })}=\left(2 m_{e} \cdot \varepsilon_{e}\right)^{1 / 2} /(e \cdot B) \approx 10^{-7} \mathrm{~m} \ll r_{(1)}, \\
r_{\mathrm{Li}}^{(\text {extr })}=\left(2 M_{i} \cdot \varepsilon_{e}\right)^{1 / 2} /(e \cdot Z \cdot B) \approx 10^{-5} \mathrm{~m} \ll r_{(1)},
\end{gathered}
$$

где $r_{\mathrm{Le}}^{(\text {extr })}$ и $r_{\mathrm{Li}}^{(\text {extr })}$ - ларморовский радиус электрона и иона на границе перетяжки. В указанных условиях замагниченные электроны и ионы дрейфуют вдоль оси разряда, обеспечивая протекание тока, что, в силу значительного различия величины ларморовского радиуса для тех и других, создает на внешней границе перетяжки тонкий (толщиной порядка радиуса Дебая) слой с избыточным положительным зарядом. Соответственно внутри перетяжки за пределами слоя плазма приобретает избыточный отрицательный заряд.

При включении механизма роста сопротивления плазмы в перетяжке, обусловленного ее турбулизацией за время $\tau_{\text {turb }} \leq 10^{-12} \mathrm{~s}[27,36]$, происходит рост напряженности электрического поля, разогрев электронной компоненты плазмы и переход электронов в режим ускорения в приосевой области. Воспользуемся следующими соотношениями для оценки характерного времени электрон-ионных столкновений и средней длины свободного пробега [31]:

$$
\begin{gathered}
\tau_{e i}=4.5 \cdot 10^{-2} \cdot T_{e}^{3 / 2} / n_{e}, \\
\lambda_{e i} \approx \tau_{e i} v_{e},
\end{gathered}
$$

где $T_{e}, n_{e}$ и $v_{e}$ - температура в $[\mathrm{K}]$, концентрация в $\left[\mathrm{cm}^{-3}\right]$ и средняя скорость электронов в плазме при температуре $T_{e}$ соответственно, а размерность $\tau_{e i}-$ [s]. Нетрудно убедиться, что при $T_{e} \approx 50 \mathrm{eV}$ имеем [31] 
$\tau_{e i} \approx \tau_{e e} \approx 2 \cdot 10^{-13} \mathrm{c}, \lambda_{e i} \approx 10^{-6} \mathrm{~m} \ll r^{(1)}$, т. е. электронная компонента плазмы, получая энергию от электрического поля, нагревается и эффективно передает энергию ионам. Уже при $T_{e} \approx 500 \mathrm{eV}$ имеем $\tau_{e i} \approx 10^{-11} \mathrm{~s}$, $\lambda_{e i} \approx 10^{-4} \mathrm{~m}$, т.е. электроны имеют возможность переходить в режим непрерывного ускорения в пределах перетяжки. Заметим, что при достижении условий для развития процесса ускорения время пролета электрона вдоль перетяжки составит $\tau_{f l y} \approx \tau_{e i}$. В свою очередь, произойдет увеличение радиуса Дебая и толщины внешнего слоя с избыточным положительным зарядом:

$$
\begin{gathered}
r_{D} \approx\left[\left(2 \varepsilon_{0} \cdot \varepsilon_{e}\right) /\left(e^{2} \cdot n_{e}\right)\right]^{1 / 2} \approx 4 \cdot 10^{-9} \mathrm{~m} \\
r_{D}^{*} \approx\left[\left(2 \varepsilon_{0} \cdot \varepsilon_{e}^{*}\right) /\left(e^{2} \cdot n_{e}\right)\right]^{1 / 2} \approx 10^{-7} \mathrm{~m}
\end{gathered}
$$

где $r_{D}$ и $r_{D}^{(*)}$ - радиус Дебая соответственно до и после развития ускорительных процессов, $\varepsilon_{e}^{(*)} \approx 10^{4} \mathrm{eV}$ - характерная энергия ускоренных электронов, $n_{e} \approx 10^{26} \mathrm{~m}^{-3}[33,34]$. Количество ионов в дебаевском слое будет составлять порядка:

$$
N_{i}^{D} \approx\left(2 \pi \cdot r^{2} \cdot r_{D}\right) / Z \sim 10^{9}-10^{11},
$$

с учетом того, что длина перетяжки принята равной ее радиусу.

С переходом электронов в приосевой области перетяжки в режим непрерывного ускорения будет происходить обеднение электронами плазмы за пределами дебаевского слоя, и она будет приближаться к квазинейтральному состоянию. А ионы в дебаевском слое испытают кулоновский взрыв. Продолжительность ухода убегающих электронов из перетяжки и продолжительность кулоновского взрыва оценим как:

$$
\begin{gathered}
\tau_{f l y}^{(*)} \approx r /\left(2 \varepsilon_{e}^{(*)} / m_{e}\right)^{1 / 2} \approx 10^{-12} \mathrm{~s}, \\
\tau_{\text {expl }} \approx r /\left(2 \varepsilon_{i} / M_{i}\right)^{1 / 2} \approx 3 \cdot 10^{-11} \mathrm{~s} .
\end{gathered}
$$

Скорость ускоренных электронов, покидающих перетяжку, более чем на порядок превышает тепловую скорость электронов, поступающих в перетяжку. Поэтому образующийся в результате явления убегания дефицит количества электронов в перетяжке оценим следующим образом:

$$
\begin{aligned}
\Delta N_{e} \approx & \pi\left(r^{(a c c)}\right)^{2} \cdot r \cdot n_{e}-\left[n_{e} \cdot\left(2 \varepsilon_{e} / m_{e}\right)^{1 / 2} / 4\right] \cdot \pi\left(r^{(a c c)}\right)^{2} \\
& \times \tau_{\text {expl }} \approx 10^{10}-10^{11} .
\end{aligned}
$$

Отметим, что результаты проведенных измерений и проделанных оценок в рамках предложенной модели не противоречат друг другу.

Мы также провели оценку соотношения плотностей энергии электрического и магнитного полей в области формирования избыточного объемного заряда, т.е. в дебаевском слое плазмы перетяжки. В рассматриваемой ситуации в силу малой толщины слоя это равноценно оценке отношения энергий указанных полей, которую использовали в качестве критерия возможности кулоновского взрыва авторы работы [5]. Оказалось, что в первом сжатии предложенный в [5] критерий не выполняется, что противоречит нашим наблюдениям.

Интересно отметить, что в рамках предложенной модели кулоновский взрыв не обязан прекращать протекание тока через перетяжку и останавливать процесс второго сжатия.

Теперь рассмотрим вопрос о том, могут ли существовать причины, препятствующие осуществлению кулоновского взрыва после второго сжатия.

В отсутствие джоулева тепловыделения и диссипативных процессов сжатие токового канала в Z-пинче магнитными силами представляло бы собой адиабатические незатухающие колебания [37]. Когда ток в разряде превышает значение Пизе-Брагинского [38,39], доминирующие лучистые потери энергии превышают джоулево тепловыделение и уменьшают энергию радиальных колебаний плазмы [13]. Присутствие в плазме перетяжки значительных радиальных колебаний имеет экспериментальное подтверждение. Пространственновременные измерения интенсивности мягкого рентгеновского излучения плазмы микропинчевого разряда, выполненные с высоким разрешением во времени и пространстве, позволили автору $[40,41]$ выявить свидетельства наличия радиальных магнитно-звуковых колебаний в перетяжке в момент наибольшего сжатия. В [42] было отмечено, что характер поляризации ионов FeXVIII-FeXIX указывает на преобладание поперечных микроскопических полей над внешним продольным электрическим полем в процессе второго сжатия. Возможно, в этих условиях убегание электронов из перетяжки отсутствует.

Выполним некоторые дополнительные оценки. Расчет характерного времени ион-ионных столкновений, т.е. продолжительности торможения (точнее потери радиально направленной скорости) ионов, совершающих поперечные колебания в плазме перетяжки после второго сжатия, согласно [31], можно приближенно выполнить следующим образом:

$$
\tau_{i i}=\left(2 M_{i} / m_{e}\right)^{1 / 2} \cdot\left(T_{i} / T_{e}\right)^{3 / 2} \cdot \tau_{i e} .
$$

Если в качестве величины температуры мы примем среднюю кинетическую энергию ионов, приобретаемую в процессе второго сжатия $T_{i} \approx\left\langle\varepsilon_{i}\right\rangle \approx 10^{4} \mathrm{eV}$, $v_{i}=\left(2\left\langle\varepsilon_{i}\right\rangle / M_{i}\right) \approx 2 \cdot 10^{5} \mathrm{~m} / \mathrm{s}, T_{e} \approx 10^{3} \mathrm{eV}$, то продолжительность торможения составит $\tau_{i i} \approx 3 \cdot 10^{-9} \mathrm{~s}$, что много больше времени второго сжатия [6], а длина свободного пробега ионов $\lambda_{i i} \approx 6 \cdot 10^{-4} \mathrm{~m}-$ много больше радиуса перетяжки, образующейся в результате второго сжатия. В этих неравновесных условиях формирование дебаевского слоя становится невозможным.

После осуществления второго сжатия происходит аномальный рост сопротивления плазмы в перетяжке и еe разогрев, сопровождающийся расширением области перетяжки [6]. В момент време- 
ни, когда электронная температура достигает величины $10 \mathrm{keV}$, оказывается, что $r_{\mathrm{Li}} \approx 2 \cdot 10^{-5} \mathrm{~m}$ и $\lambda_{e e} \approx \lambda_{i i} \approx 10^{-1} \mathrm{~m} \gg r \approx 10^{-4} \mathrm{~m} \gg r_{D} \approx 10^{-8} \mathrm{~m}$, где $r$ - радиус перетяжки в указанный момент времени. Появляется возможность формирования дебаевского слоя. Напряженность квазистатического электрического поля резистивной природы можно оценить величиной

$$
E_{\text {res }}^{(2)} \approx I \cdot R / r_{(2)} \approx 10^{11} \mathrm{~V} / \mathrm{m},
$$

где $R \approx 10 \Omega$ - сопротивление плазмы перетяжки на стадии завершения второго сжатия [30], $r_{(2)} \sim 10^{-5} \mathrm{~m}-$ радиус пинча на стадии второго сжатия. Оценка по формуле (19) выполнена в предположении, что практически весь разрядный ток $I \approx 10^{5}$ А протекает через зону сжатия. Если положить количество электронов в дебаевском слое с учетом падения концентрации частиц примерно на 3 порядка величины (вследствие разлета плазмы из микропинча после второго сжатия) равным:

$$
N_{e} \approx 2 \pi r^{2} \cdot r_{D} \cdot n_{e} \approx 10^{8}-10^{9},
$$

то напряженность кулоновского поля нескомпенсированного положительного заряда ионов составит

$$
E_{c o l} \approx\left(e \cdot N_{e}\right) /\left(4 \pi \varepsilon_{0} \cdot r^{2}\right) \approx 10^{9}-10^{10} \mathrm{~V} / \mathrm{m} \ll E_{r e s} .
$$

Таким образом, в процессе нагрева и одновременного расширения плазмы перетяжки после второго сжатия, условия для реализации кулоновского взрыва, по всей видимости, отсутствуют.

\section{Заключение}

Экспериментально обнаружены свидетельства возможной реализации „кулоновского взрыва“ в области формирования микропинча в разряде типа Z-пинч в среде тяжелых элементов вследствие убегания ускоренных в осевом направлении высокоэнергетичных электронов. Энергия зарегистрированных ионов $1-3 \mathrm{MeV}$ соответствует величине, достижимой в результате кулоновского взрыва дебаевского слоя в плазме перетяжки на стадии, предшествующей радиационному сжатию и образованию „Горячей точки“6 в микропинче.

\section{Конфликт интересов}

Авторы заявляют, что у них нет конфликта интересов.

\section{Список литературы}

[1] G. Benford. Appl. Phys. Lett., 33 (10), 983 (1978).

[2] В.В. Вихрев, Е.О. Баронова. Прикладная физика, 5, 71 (1999).

[3] Б.А. Трубников, С.К. Жданов, В.П. Власов. Физика плазмы, 17 (10), 1192 (1991).

[4] А.А. Рухадзе, У. Юсупалиев. ЖТФ, 74 (7), 127 (2004).

[5] В.И. Орешкин, Е.В. Орешкин. ЖТФ, 87 (1), 34 (2017).
[6] В.В. Вихрев, В.В. Иванов, К.Н. Кошелев. Физика плазмы, $8(6), 1211$ (1982).

[7] С.И. Блинников, В.С. Имшенник. Физика плазмы, 8(1), 193 (1982).

[8] А.Н. Долгов, Н.А. Клячин, Д.Е. Прохорович. ЖТФ, 84 (12), 145 (2014)

[9] Э.Я. Гольц, И.А. Житник, Э.Я. Кононов, С.Л. Мандельштам, Ю.В. Сидельников. ДАН СССР, 200 (3), 560 (1975).

[10] В.А. Веретенников, С.Н. Полухин, О.Г. Семенов, Ю.В. Сидельников. Физика плазмы, 7 (6), 1199 (1981).

[11] А.Н. Долгов, Д.Е. Прохорович. Прикладная физика, 3, 52 (2008).

[12] А.Н. Долгов, Н.А. Клячин, Д.Е. Прохорович. Физика плазмы, 38 (2), 168 (2012).

[13] J.W. Shearer. Phys. Fluids, 19 (9), 1426 (1976).

[14] J. Bailey, A. Fisher, N. Rostoker. J. Appl. Phys., 60 (6), 1939 (1986).

[15] К.Н. Кошелев, Ю.В. Сидельников, В.В. Вихрев, В.В. Иванов. Спектроскопия в горячей плазме многозарядных ионов (Наука, М., 1991), с. 163.

[16] В.И. Орешкин. Изв. вузов. Физ., 40 (12), 76 (1997).

[17] L. Bernal, H. Bruzzone. Plasma Phys. Contr. Fusion, 44 (2), 223 (2000).

[18] А.Н. Долгов. Эмиссия частии и излучения в микропинчевом разряде (Дисс. докт. физ.-мат. наук. М., 2005), $232 \mathrm{c}$.

[19] В.А. Веретенников, В.А. Грибков, Э.Я. Кононов, О.Г. Семенов, Ю.В. Сидельников. Физика плазмы, 7 (2), 455 (1981).

[20] В.А. Веретенников, А.Н. Долгов, О.Н. Крохин, О.Г. Семенов. Физика плазмы, 11 (8), 1107 (1985).

[21] А.Н. Долгов, В.К. Ляпидевский, Д.Е. Прохорович, А.С. Савелов, Г.Х. Салахутдинов. Физика плазмы, 31 (2), 192 (2005).

[22] T. Czyzwski, A. Szydlovski. Jour. Tech. Phys., 22 (22), 153 (1981).

[23] W.A. Stygar, R.J. Leeper, L.P. Mix, E.R. Brock, J.E. Bailey, D.E. Hebron, D.J. Johnson, T.R. Lockner, J. Maenchen, T.A. Mehlhorn, P. Reyes. Rev. Sci. Instr., 59 (8), 1703 (1988).

[24] Г.В. Иваненков, С.А. Пикуз, Д.Б. Синарс, В. Степневски, Д.А. Хаммер, Т.А. Шелковенко. Физика плазмы, 26 (10), 927 (2000).

[25] С.А. Пикуз, Т.А. Шелковенко, Д.А. Хаммер. Физика плазмы, 41 (4), 319 (2015).

[26] V.I. Oreshkin, S.A. Chaikovsky, A.P. Artyomov, N.A. Labetskaya, A.V. Fedunin, A.G. Rousskikh, A.S. Zhigalin. Phys. Plasmas, 21, 102711 (2014).

[27] В.В. Аверкиев, А.Н. Долгов, В.К. Ляпидевский, А.С. Савелов, Г.Х. Салахутдинов. Физика плазмы, 18 (6), 724 (1992).

[28] А.Н. Долгов, Н.А. Клячин, Д.Е. Прохорович. Физика плазмы, 42 (12), 24 (2016).

[29] А.Н. Долгов, Н.А. Клячин, Д.Е. Прохорович. Письма в ЖЭТФ, $99(9), 595$ (2014).

[30] А.Н. Долгов, В.В. Вихрев. Физика плазмы, 31 (3), 290 (2005).

[31] С.К. Жданов, В.А. Курнаев, М.К. Романовский, И.В. Цветков. Основы физических прочессов в плазме и плазменных установках (МИФИ, М., 2007)

[32] B.L. Weich, F.C. Young, H.R. Griem. J. Appl. Phys., 74 (4), 2260 (1993).

[33] Г.С. Саркисов, Б. Этлишер. Письма в ЖЭТФ, 62 (9-10), 775 (1995). 
[34] В.А. Веретенников, А.Е. Гурей, Т. Писарчик, С.Н. Полухин, А.А. Рупасов, Г.С. Саркисов, О.Г. Семенов, А.С. Шиканов. Физика плазмы, 16 (7), 818 (1990).

[35] А.Н. Долгов, Н.В. Земченкова, Н.А. Клячин, Д.Е. Прохорович. Физика плазмы, 37 (3), 194 (2011).

[36] С.М. Захаров, Г.В. Иваненков, А.А. Коломенский, С.А. Пикуз, А.И. Самохин. Физика плазмы, 9 (3), 469 (1983).

[37] Б.Э. Мейерович. Канал сильного тока (ООО ФИМА, M.,1999)

[38] R.S. Pease. Proc. Phys. Soc., B70 (445), 11 (1957).

[39] С.И. Брагинский. ЖЭТФ, 33 (9), 645 (1957).

[40] Б.Н. Миронов. Физика плазмы, 20 (6), 546 (1994).

[41] Б.Н. Миронов. Физика плазмы, 20 (10), 886 (1994).

[42] А.Н. Долгов, Н.А. Клячин, Д.Е. Прохорович. Физика плазмы, 40 (9), 836 (2014). 